\title{
In situ Methods for Analysis of Polymer Electrolyte Membrane Fuel Cell Materials by Soft X-ray Scanning Transmission X-ray Microscopy
}

\author{
Adam P. Hitchcock ${ }^{1}$, Viatcheslav Berejnov ${ }^{2}$, Vincent Lee ${ }^{1}$, Darija Susac ${ }^{2}$ and Juergen Stumper ${ }^{2}$ \\ 1. Dept. of Chemistry \& Chemical Biology, McMaster University, Hamilton, Canada \\ ${ }^{2}$ Automotive Fuel Cell Cooperation, Burnaby, Canada
}

The performance, efficiency and lifetime of polymer electrolyte membrane fuel cells (PEM-FC) is a function of the composition and nanostructure of the electrodes. Optimization of these properties is critical, in particular for the cathode where the rate limiting oxygen reduction reaction takes place. For the past 5 years we have used soft X-ray scanning transmission X-ray microscopy (STXM) to study a wide range of issues in PEM-FC optimization [1-5]. Most of these studies have been carried out on dry, microtomed samples at ambient temperature $(25 \mathrm{C}, 0 \% \mathrm{RH})$ which are very different from the typical operating conditions of the PEM-FC materials $(70 \mathrm{C}, 80 \% \mathrm{RH})$. It is known that there are significant changes to the nanostructure with temperature and between dry and hydrated conditions. Thus instrumentation and methods to examine focused ion beam (FIB) or microtomy thin sections of PEM-FC membrane electrode assemblies (MEA) under more realistic conditions is needed. The in situ approach would be used to control water saturation in cathode and membrane, apply temperature changes and visualize the response of the PEM-FC materials in freeze-thaw cycling. The ability to change the sample environment in situ while examining the consequences will give added value to STXM investigations of PEM-FC. In situ STXM can be applied to many other areas of science, such as aerosol research [6].

STXM uses tunable synchrotron radiation, zone plate focusing and near edge X-ray absorption (NEXAFS) spectral contrast for speciation, and quantitative mapping of chemistry in 2-d and 3-d with sub $30 \mathrm{~nm}$ spatial resolution [7]. As a photon-in, photon-out technique, it is well suited for in-situ materials, environmental and biological studies. The phase, amount and spatial distribution of water in MEAs can be determined by $\mathrm{O} 1 \mathrm{~s}$ measurements since gas, liquid and solid water are readily differentiated by their O 1s NEXAFS spectra (Fig. 1 ). We have designed, built and are commissioning an apparatus for in situ environmental modification, with targets of -30 to $+80 \mathrm{C}$ and $0-100 \% \mathrm{RH}$ (Fig. 2). To date we have achieved conditions of (-16 C, water saturation) and (+45 C, $50 \%$ RH). Fig. 3 presents results for chemical mapping of water in a PEM-FC under various conditions, ranging from dry, ambient, to $5 \mathrm{C}, 100 \% \mathrm{RH}$, to $-16 \mathrm{C}$, saturated, with evidence of ice formation. A significant issue for reliability of automotive fuel cell applications in cold climates is potential damage if water freezes in the MEA nanostructure. The in situ STXM environmental cell offers a means to compare the susceptibility of different MEAs to ice formation. The environmental cell instrumentation, calibration by deliquescence and efflorescence transitions in alkali halide salts, and its present performance will be described, along with results of recent PEM-FC environmental studies [8].

\section{References:}

[1] V. Berejnov, Z. Martin, M.M. West, S. Kundu, D. Bessarabov, J. Stumper D. Susac and A.P. Hitchcock, Probing platinum degradation in polymer electrolyte membrane fuel cells by scanning transmission X-ray microscopy, Phys. Chem. Chem. Phys. 14 (2012) 4835.

[2] D. Susac, V. Berejnov, A.P. Hitchcock, and J. Stumper, STXM Study of the Ionomer Distribution in PEMFuel Cell Catalyst Layers, ECS Transactions 41 (2011) 629. 
V Berejnov, D. Susac, J. Stumper and A.P. Hitchcock, 3D Chemical Mapping of PEM Fuel Cell Cathodes by Scanning Transmission Soft X-ray Spectrotomography, ECS Transactions, 50 (2012) 361.

[3] D. Susac, V. Berejnov, A.P. Hitchcock and J. Stumper, STXM Characterization of PEM Fuel Cell Catalyst Layers, ECS Transactions 50 (2012) 405.

[4] V. Berejnov, D. Susac, J. Stumper and A.P. Hitchcock, Nano to Micro Scale Characterization of Water Uptake in The Catalyst Coated Membrane Measured by Soft X-ray Scanning Transmission X-ray Microscopy, ECS Transactions 41 (2011) 395.

[5] S.T. Kelly, P. Nigge, S.Prakash, A.Laskin, B. Wang, T. Tyliszczak, SR. Leone, and M. K. Gilles, An environmental sample chamber for reliable scanning transmission $x$-ray microscopy measurements under water vapor Rev. Sci. Inst. 84 (2013) 073708.

[6] A.P. Hitchcock, Soft X-ray Imaging and Spectromicroscopy in Handbook on Nanoscopy, eds. G.Van Tendeloo, D. Van Dyck and S.J. Pennycook (Wiley, 2012) 745.

[7] This research is supported by NSERC, CFI, OIT, AFCC and Canada Research Chair funding. CLS is supported by NSERC,CIHR, NRC and U. Saskatchewan. ALS (LBNL) is supported by BES, DOE.

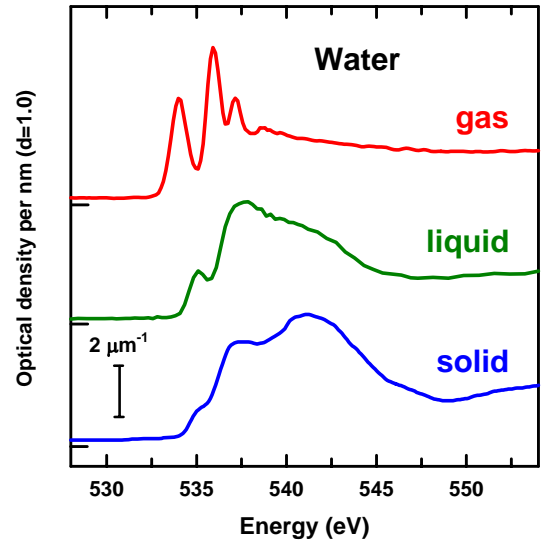

Fig. 1 O 1s spectra of gas, liquid and solid water. Intensity scale is absolute $(\mathrm{OD} / \mathrm{nm})$. The density for all 3 phases was set at $1.0 \mathrm{~g} . \mathrm{cm}^{-3}$.
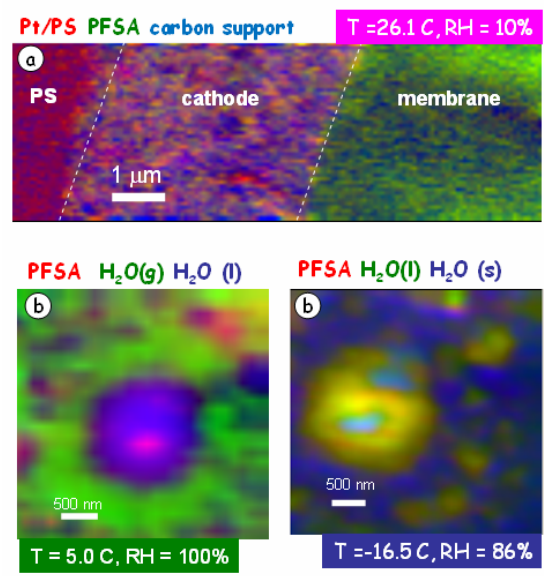

Fig. 3 Color coded composites (rescaled) of component maps derived from fits of $\mathrm{O} 1 \mathrm{~s}$ image sequences to reference spectra for perfluorosulfonic acid (PFSA), carbon support, gas, liquid and solid water. (a) overview of cathode - membrane region of a MEA at $26 \mathrm{C}$, $10 \% \mathrm{RH}$. (b) water nucleation region in membrane at $5 \mathrm{C}$, $100 \% \mathrm{RH}$ (c) ice nucleation region at $-16.5 \mathrm{C}, 86 \% \mathrm{RH}$ (CLS).
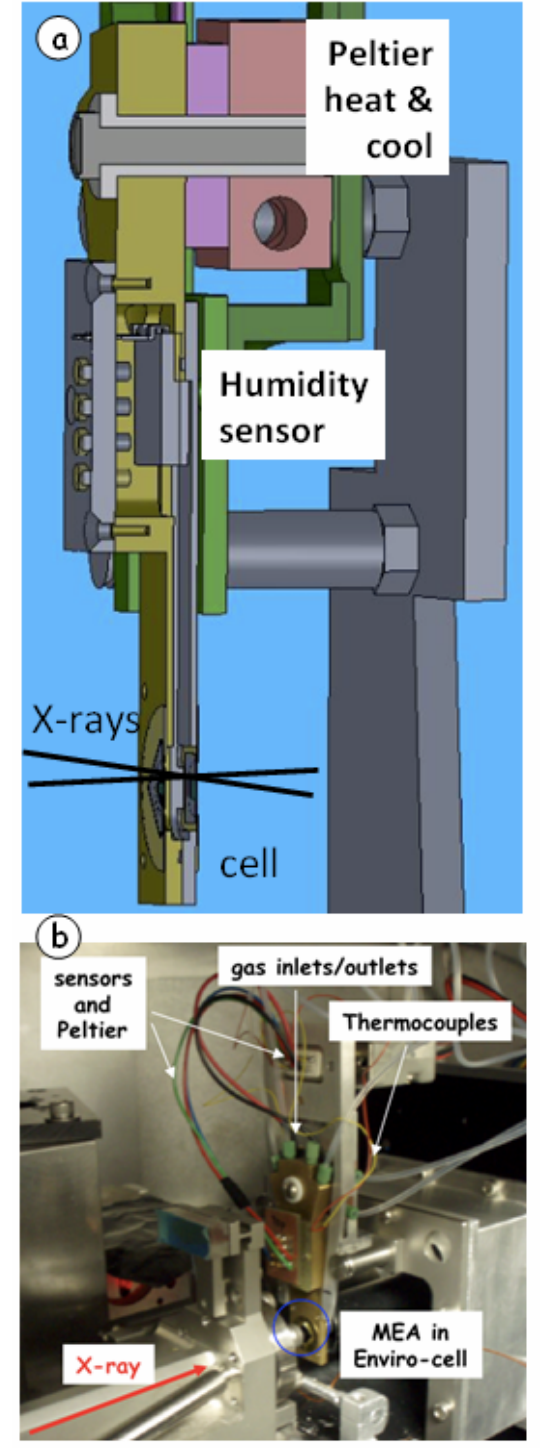

Fig. 2 . (a) CAD cartoon, and (b) photograph of the variable temperature, variable humidity environmental cell in STXM 5.3.2.2 (ALS). 